EGU21-13151, updated on 18 May 2021

https://doi.org/10.5194/egusphere-egu21-13151

EGU General Assembly 2021

(c) Author(s) 2021. This work is distributed under

the Creative Commons Attribution 4.0 License.

\title{
New sights in crenulation geometry developed in anisotropic materials undergoing simple shear deformation
}

\author{
Yuanbang Hu $\mathbf{u}^{1,2}$, Tamara de Riese ${ }^{1}$, Paul Bons ${ }^{1}$, Shugen Liu ${ }^{2}$, Albert Griera ${ }^{3}$, Maria-Gema Llorens ${ }^{4}$, \\ Enrique Gomez-Rivas ${ }^{5}$, and Melanie Finch ${ }^{1,6}$ \\ ${ }^{1}$ Department of Geosciences, Eberhard Karls University Tübingen, Tübingen, Germany \\ ${ }^{2}$ College of Earth Science, Chengdu University of Technology, Chengdu, China \\ ${ }^{3}$ Departament de Geologia, Universitat Autònoma de Barcelona, Barcelona, Spain \\ ${ }^{4}$ Institute of Earth Sciences Jaume Almera (ICTJA-CSIC), Barcelona, Spain \\ ${ }^{5}$ Department of Mineralogy, Petrology and Applied Geology, University of Barcelona, Barcelona, Spain \\ ${ }^{6}$ School of Earth, Atmosphere and Environment, Monash University, Clayton, Victoria, Australia
}

Deformation of foliated rocks commonly leads to crenulation or micro-folding, with the development of cleavage domains and microlithons. We here consider the effect of mechanical anisotropy due to a crystallographic preferred orientation (CPO) that defines the foliation, for example by of alignment of micas. Mechanical anisotropy enhances shear localisation (Ran, et al., 2018; de Riese et al., 2019), resulting in low-strain domains (microlithons) and high-strain shear bands or cleavage domains. We investigate the crenulation patterns that result from moderate strain simple shear deformation, varying the initial orientation of the mechanical anisotropy relative to the shear plane.

We use the Viscoplastic Full-Field Transform (VPFFT) crystal plasticity code coupled with the modelling platform ELLE (http://www.elle.ws; Llorens et al., 2017) to simulate the deformation of anisotropic single-phase material with an initial given CPO in dextral simple shear in low to medium strain. Deformation is assumed to be accommodated by glide along the basal, prismatic and pyramidal slip systems of a hexagonal model mineral. An approximately transverse anisotropy is achieved by assigning a small critical resolved shear stress to the basal plane. An initially point-maximum CPO at variable angles to the shear plane defines the initial straight foliation at different angles to the shear plane, limiting ourselves to orientations in which the foliation is in the stretching field. The resulting crenulation geometries strongly depend on the orientation of the foliation and we observe four types of localisation behaviour: (1) synthetic shear bands, (2) antithetic shear bands, (3) initial formation of antithetic shear bands and subsequent development of synthetic shear bands, and (4) distributed, approximately shear-margin parallel strain localisation, but no distinct shear bands.

The numerical simulations not only show the evolving strain-rate field, but also the predicted finite strain pattern of existing visible foliations. We show the results for layers parallel to the foliation, but also cases where the visible layering is at an angle to the mechanical anisotropy (e.g. in case of 
distinct sedimentary layers and a cleavage that controls the mechanical anisotropy). A wide range of crenulation types form as a function of the initial orientation of the visible layering and mechanical anisotropy (comparable to C, C' and C" shear bands and compressional crenulation cleavage). Most importantly, some of may be highly misleading and may easily be interpreted as indicating the opposite sense of shear.

\section{Reference}

de Riese, T., et al. (2019). Shear localisation in anisotropic, non-linear viscous materials that develop a CPO: A numerical study. Journal of Structural Geology, 124, 81-90. DOI: 10.1016/j.jsg.2019.03.006

Llorens, M.-G., et al. (2017). Dynamic recrystallisation during deformation of polycrystalline ice: insights from numerical simulations. Philosophical Transactions of the Royal Society A, Special Issue on Microdynamics of Ice, 375: 20150346. DOI: 10.1098/rsta.2015.0346.

Ran, H., et al. (2018). Time for anisotropy: The significance of mechanical anisotropy for the development of deformation structures. Journal of Structural Geology, 125, 41-47. DOI: 10.1016/j.jsg.2018.04.019 